Tordecilla $d$ y cols.

Rev, Chil. Pedlatr. 64 (1); 36-39, 1993

\title{
Carcinoma nasofaríngeo en la infancia
}

\author{
Juan Tordecilla C. ${ }^{1}$; Myriam Campbell B. ${ }^{1}$; Ricardo Pérez C. ${ }^{2}$
}

\section{Nasopharyngeal carcinoma in childhood}

A 13 yeat ded girl was seen because of persistent upper respiratory symptoms and bilateral cervicai lymph node enlargenent Needle aspiration of o right pharyngeal tumor, biosy, compuled axial tomography, thorax and bone $x$ roy films were compatible with a stoge IV nosopharygeal corcinoma. Serological evidence of previous infection by Epstein Barr virus was also laund. Some lymph node enlargement persisted after appropriate Iadialion therapy, but good subsequent response to chemotheraphy was observed. This malignancy has low incidence in pediafric patients. Diagnosis is bosed an computed oxial tomogrophy of the nosophoringeal region and biopsy of tumoral mass or involved tymph nodes. There is a frequent associalion with previous Epslein Barr virus infection. Prognosis depends on clinical stoge of the disease and current trealment consist in iradiation and chemotheropy.

(Key words: Nasopharingeal neoplasm, carcinoma, Ebstein Barr virus.)

El carcinoma nasofaríngeo (CNF) es una neoplasia primaria del epitelio nasofaríngeo que tiene una baja frecuencia en los nifos 1.2 con marcadas diferencias geograficas en su incidencia ${ }^{3} y$ wa conocida asociación con la infección por el virus de Epstein Barr.4. Clínicamente se caracteriza por adenopatía cervical producto de la metástasis inicial, obstrucción nasal, rinolalia e hipoacusia unilateral secundaria al compromiso de la trompa de Eustaquio 5.6 . El pronóstico de la enfermedad depende del estadio clínico, con sobrevida a 5 años entre 30 a $60 \%$ ? Se comunica un caso clínico con el propósito de contribuir al conocimiento de esta afección ante la escasez de experiencia sobre ella en nuestro medio ${ }^{8}$.

\section{Caso clínico}

Niña de 13 aćos consulto en septiembre de 1991, en el policlínico de hematología del Hospital Robeno del Rio, por aumento de volumen ganglionar cervical bilateral de 5 a $6 \mathrm{~cm}$ de diámetro, firme, sensible, de un mes de evolución, usociado a coriza, disfonía y odinofagia, sin fiebre (figura 1). No se encontrarou ruidos pulmonares agregados ni hepatesplenomegalia. Hemograma, radiografía de tórax y monotest normales. Se le indicaton antibióticos $\sin$ observar

1. Unidad de Hematología-Oncología, Hospjtal Robeno del Río.

2. Servicio Radjoterapia, Instituto Caupolicán Pardo Correa.

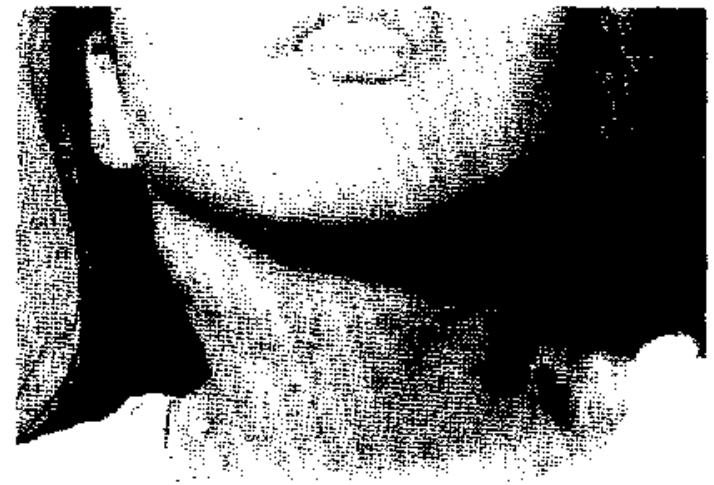

Figura 1: Aumento de volumen ganglionar submaxilar y cervical bilateral.

respuesta a los 15 días. Un mes después, por no existir cambios en el tamaño de los ganglios y agregarse desviación de la úvula hacia la izquierda, con desplazamiento de la amígdala derecha en sentido medial, se efectúo punción ganglionar de la que se obuvieron células de características malignas. Una tomografía axial computarizada ratific $\delta$ la presencia de masa tumoral en la zona nasofaringea y ganglios linfáticos bilaterales, sin compromiso de la base del cráneo (figura 2). La biopsia de la masa nasofajingea fue informada como carcinoma indiferenciado (linfoepiteliomas). E cintigrama óseo y el examen químico y citológico del líquido cefalorraquídeo fueron normales. El estudio de inmunoglobulina $\mathbf{G}$ antivins de Epstein Barr fue posilivo. I_a paciente se elspificó de acuerdo al ctiterio de "A merican Joint Commiuee on Csncer" (AJC) y qued 6 catalogada como $T$ (Tumor) $3 \times$ (Adenopatía) $3 b$ y M (Metástasis), estadio IV. Se trato con radioterapia a dosis de 7000 rads al tumor 


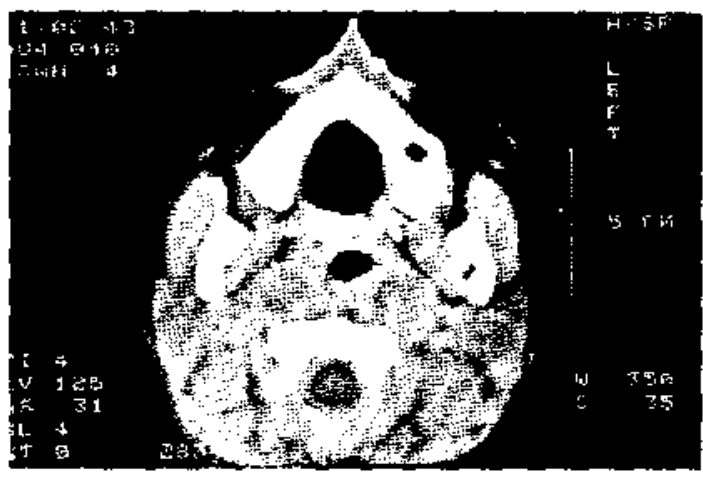

Figura 2: Tomograffa axial al diagnóstico con masa tumotal desde zona nasofaríngea hasta ganglios cervicales.

primario, $5500 \mathrm{rads}$ en el cuello, total, con protección medular a los 4500 , más sobreimpresión a las adenopatías cervicales hasta alcanzar los 7000 rads. Al término de esta Icrapia, en octubre de 1991, se constató persistencia de adenopatía cervical derecha, por lo que se inició quimioicrapia con ciclos de vincristina-adriamicina-ciclofosfamida altemando cada 21 días con metotrexato-cisplatino-5. fluoruracilo en jufusion continua de 5 días. Durante este tratamiento la paciente presentó dos episodios de ncutropenia febril y nucositis severa. La respuesta clínica fue satisfacloria, en enero de 1992, una lomografía axial de control (figura 3) revelo que, prácticamente, habian desaparecido la masa tumotal y las adenopatías.

Actualmente está en buenas condiciones. No hay adenopatías. La evaluación enđocrinológica no detectó alteraciones tiroidcas. El estudio otorrinolaringologico es normal, pero bajó 8 kilos de peso y presenta fibrosis de la zona cervical irtadiada, Sigue en trazamiento quimioterápico planíficado por un año.

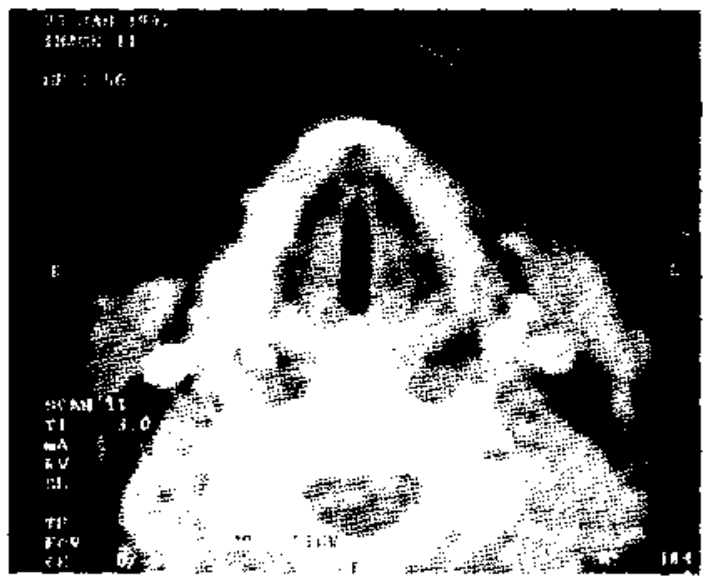

Figurn 3: Tomografía axial de control loego de radio y quimioterapia que muestra regresión de masas tumorales.

\section{Comentario}

La incidencia del carcinoma nasofaríngeo en nifios es de 1 por 100000 niños < 15 aflos en EUA y de 15 a 20 por 100000 en cl sureste de Asia y en el norte de Africa, donde constituye la sexta causa de muerte por cáncer en la población menơ de 30 años'. Es más frecuente en hombres y ticne su mayor incidencia en la segunda década de la vida. Se han reportado casos familiares. Fue descrito separadamente por Regaud y Schmincke en 1921 y se trata de un carcinoma epidermoide indiferenciado, originado generalmente en el anillo de Waldeyer. Existen dos modelos histológicos: el tipo Regaud, con colecciones bien definidas de células epileliales alrededor de los linfocitos y el tejido conectivo y el tipo Schmincke, en el que las células tumorales están difusamente distribuidas y entremezcladas con las células inflamatorias ${ }^{10,11}$. Clínicamente, como en nuestra paciente, la adenopatía cervical, es un hecho central, presente en $95 \%$ de los casos, de preferencia en las cadenas cervical posterior y yugular ${ }^{12}$. Frente a una adenopatía cervical, dura, indolora y de larga evolución debe realizarse un cuidadoso examen de la cabeza y cuello en busca del tumor primario, ya que un alto porcentaje de estas masas son metástasis regionales ${ }^{13-15}$.

El examen otorrinolaringológico es imperativo en estos casos, pues permite reconocer un alto porcentaje de tumores primarios de rinofaringe, orofaringe, hipofaringe y laringe supraglótica. Existe una relación indirecta entre el tumor primario (T) y la adenopatia (N) y en ocasiones, en que el examen otorrinolaringológico es negativo, puede ser necesaria la biopsia a ciegas de las regiones mencionadas. A la inversa, en boca, nariz y cavidades perinasales la relación entre tumor y adenopatía es directa. El trismus en los niños es infrecuente, pero, en el carcinoma nasofaringeo ocurre en más de $50 \%$ de los casos. También puede haber otalgia, sordera, coriza, epistaxis y parálisis de los nervios craneales por compromiso de la base del crắneo ${ }^{16-18}$.

Cuando se sospecha carcinoma nasofaríngeo en un niño se recomienda bacer examen cuidadoso otorinolaringologico de la región rinofaríngea y ótica en busca de compromiso de la trompa de Eustaquio; rastreo de metástasis con radiografia de tórax y cintigrama óseo; tomografía axial computadorizada de nasofaringe para observar si hay compromiso de la base del cráneo; 
búsqueda de células malignas en el líquido cefalorraquídeo; biopsia de la masa nasofaríngea $y$ de los ganglios cervicales'?

La clasificación más ampliamente aceptada de estos tumores es la de AJC basada en el tamaño del tumor primario (T), las adenopatías (N) y la presencia de metástasis (M); en los países asiáticos se usa además una modificada por $\mathrm{Ho}^{20.21}$. En nuestra paciente el tumor se había extendido a la orofaringe, tenía compromiso ganglionar bilateral mayor de $6 \mathrm{~cm}$ pero no se encontraron metástasis por lo que se etapificó en estadio IV.

El virus Epstein Barr se asocia regularmente con el carcinoma nasofaringeo y la búsqueda de anticuerpos es útil en el diagnóstico. Existe elevación de anticuerpos contra el antígeno capsular. Los anticuerpos al componente difuso del antígeno viral son de mayor valor diagnóstico ${ }^{4}$. En el momento del diagnóstico se encuentran títulos altos de estos anticuerpos en 76 a $98 \%$ de los casos. Los títulos se correlacionan con el tamaño y la actividad tumoral; en los pacientes con recurrencia o metástasis hay elevación previa de êstos, lo que son útiles para el control y seguimiento. En alrededor de $30 \%$ de los casos de carcinoma nasofaríngeo oculto se encuentran títulos altos de anticuerpos para el antígeno capsular viral ${ }^{22}$. También se ha encontrado relación entre el título de anticuerpos al virus y el tipo histológico del tumor, puesto que aquéllos están francamente altos en los casos de carcinoma epidermoide no queratinizado y en el indiferenciado, pero no en el carcinoma epidermoide queratinizado4. Los métodos serológicos usuales para detoctar infección aguda por el vinus, como los anticuerpos heterófilos del monotest, no son adecuados. En nuestro paciente la IgG fue positiva y el monotcst negativo. También se ha encontrado que $34 \%$ de los pacientes con carcinoma nasofaringeo son portadores de antígenos de superficie para la hepatitis B, asociación que no ha sido aclarada del todo, pero en los pacientes en que ocurre, la sobrevida más corta9.

El diagnóstico diferencial del carcinoma nasofaríngeo incluye otras neoplasias de la misma región, incluyendo rabdomiosarcoma (que cn los niños es el cáncer más frecuente de esta zona), linfoma no Hodgkin (Burkit), carcinoma tiroídeo y neuroblastoma (estesioneuroblastoma) ${ }^{23}$.

En términos generales la terapia primaria para el carcinoma nasofaríngeo es la radioterapia. En los últimos antos se ha obtenido mejor sobrevida, empleando nuevas técnicas, que incluyen mayor dosis, más fraccionada y campos más amplios de radiación, extendiendolos al cuello en forma electiva. La dosis varía entre 6000 y 7000 rads al tumor primario, base del cráneo y los ganglios comprometidos, con fracciones diarias de 180 a 200 rads. Con estas dosis, dependiendo del estado clínico, se obtienen remisiones completas en 70 a $85 \%$ de los casos y parciales en 9 a $12 \%$ según diferentes autores ${ }^{24,} 25$. Se han visto recurrencias locales y aparición de metástasis en huesos, pulnones e hígado en pacientes tratados sólo con radioterapia, que parece insuficiente en casos de enfermedad avanzada. Por lo anterior, para mejorar el control local y evitar las metástasis a distancia, actualmente tiende a usarse quimioterapia previa, asociada o después de la radioterapia $^{26}$. Varios agentes tienen efecto sobre el tumor, especialmente cisplatino, 5-fluoruracilo, metotrexato, adriamicina, bleomicina, vincristina y VP-1627. En los pacientes con enfermedad recurrente, la quimioterapia asociada a la radioterapia tiene un rol fundamental 28 y una serie de trabajos clínicos apoyan su empleo en esta afección. Especial consideración merece la asociación de cisplatino a dosis alta y 5-fluoruracilo en infusión continua durante 5 días, que produce $90 \%$ de respuestas objelívas en pacientes en estados avanzados ${ }^{29}$. La resección quirúrgica del tumor es una opción que debe considerarse en algunos $\operatorname{casos}^{30}$. Como complicaciones del tratamiento se ha observado xerostomía y sialoadenitis, más lardíamente fibrosis y atrofía muscular del cueIlo. También se describe hipotiroidismo y la aparición de un segundo cáncer corno linfoma no Hodgkin, carcinoma pancreático y tumor de mama.

\section{Resumen}

Una paciente de 13 años, de sexo femenino, consultó por adenopatía cervical bilateral, coriza y disfonía de un mes de duración, sin fiebre ni otras manifestaciones, no respondió a tratamiento empírico con antibióticos, agregándose luego desplazamiento de la úvula y prominencia de la amígdala derecha. La punción, la biopsia, las radiografías de tórax y huesos y la tomografía axial demostraron que se trataba de un carcinoma nasofaríngeo en estadio IV, las pruebas serológicas fueron positivas para infección previa por 
virus Epstein Barr. La nifia fue tratada con radioterapia con buenos resultados, pero tuvo una adenopatía persistente, la que respondió bien a la quimioterapia posterior. Esta neoplasia tiene una baja incidencia en la edad pediátrica, clínicamente se presenta con adenopatías cervicales de larga evolución y síntomas respiratorios altos persistentes.

(Palabras clave: Adenopatía cervical persistente, neoplasia nasofaríngea, carcinoma, virus de Epstein Barr.)

\section{Referencias}

1. Fernandez CH, Cangir A, Samaan NA, Rivera R: Na. sopharyngesl carcinoma in children. Cancer 1976; 37 : 2787-2791.

2. Devasch $\boldsymbol{M}$, Mercado $R$, Parsons JA: Cancer of the nasopherynx in children. Cancer 1978; $41: 1128-1133$.

3. Gasrpar $H$, Wilmes E, WolfH: Epiderniologic, eliologic and immunologic aspects of nascpharyngeal carcinoma. I Med 1981; 12: 257-284.

4. Pearson GR, Weiland LH, Neet HB, et al.: Application of Epstein-Barr vinus (EBV) serology to the diagnosis of North American nasopharyngeal carcinoma. Cancer 1983; 51:260-268.

5. Roper HP, Essex-Cater A, Marsden HB, Dixon PF. Campbell RH: Nasopharyngeal carcinoma in children. Pedialr Hematol Oncol 1986; 3: 143-152.

6. Fonseca $X$, Guerrero $L$ : Cancer de rinofatinge. Rev Chil Onorrinolaringol 1979: 39: 7.15.

7. Baker SR, Wolfe RA: Prognostic factors of nasopharyngeal malignancy. Cancer 1982; 49; 163-169.

8. Las Herizs $J$, Osorio M, Advis P, Kakarieka E: Carcinoma Nasofarfngeo en la infancia. Rev Chil Pediatr 1984: 55: 42.46

9. Chang AY, Su SW, Zen SH, Wang WC: Nasopharyngeal carcinoma in young patients. Am $\mathrm{I}$ Clin Oncol 1991: 14: 1-4.

10. Pratt CB, Douglas EC: Management of less common cancers of childhood. In: Pizzo PA, Poplack DG: Principles and practice of Pediatric Oncology. I.B. Lippincott Company $1989 ; 759.782$.

11. Coffin CM, Rich SS, Dehner $M$ : Familial aggregation of nasopharyngeal carcinoma and other malignancies. Cancer 1991; 68: 1323-1328.

12. Linberg $R$ : Distribution of cervical lymph node metastasis from squamous cell carcinoma of the upper respintory digestive tracls. Canoer 1972; 29 : 1446 . 1451.

13. Skandalakis J, Gray $S$, Takakis $N$, Godwin J, Paer D: Tumors of the neck. Surgery 1960; 48: 375-384.
14. Celedón C: Estudio anatomoclínico de tumores cervicales. Rev Chil Otorinolaningol 1975; 35: 87-92.

15. Slawgther D, Majarakis ID. Sowthwich HW: Clinical evaluation of swelling in the neck. Surg Clin North An 1956: 36: 3-9.

16. Matalón $C$, Emmerich $E$, Scott $C$ : Tumores malignos del rinofarinx. Rev Chil Otorrinolaringol 1988; 48: 18-21.

17. Pick T, Maurer HM, McWilliams NB: Lymphoepithelioma in childhood, J. Pediatr 1974; 84:96-100.

18. Bleichner JC, Ragsdale B, Harimann DP, Merida $M$ : Nasopharyngeal malignancies in children. Ear Nose Throat J 1987; 66: 148-153.

19. Stevents $M E$, Scholer RE, Harnsbergeer $H R$; Nasopharyngeal cancer in childhood. Ear Nose Throat J 1987; 66: 104-106.

20. Manual for Staging of Concer. $2^{4}$ edition. American Joint Conmittee on Cancer. Philadelphia: J.B. Lippincontt. 1983.

21. Ho JHC: Stage classification of nasopharyngeal carcinoma: A review. In the G, Ito $Y$ (eds): Nasopharyngeal carcinoma: Etiology and control. Lyon: Intema. tional Agency for research in cancer. 1077; 99-113.

22. Yong-Sheng Z, Schan JS, Xing-Tai $O$, el al: Immunoglobulin A against viral capsid antigen of EpsteinBarr virus and indirect mirroz exarnination of the nasopharynx in the detcction of asymptomatic nasopharyngesl carcinoma. Cancer 1992; 69: 3-7.

23. Cangir A: Miscellaneous childhood tumors. In Fembach DJ, Vietti TJ: Clinical Pediatric Oncology. 4th edition. Mosby - Yeat Book, Inc. 1991; 627-645.

24. Jenkin RD, Anderson $J R$, Jereb $B$, et al.: Nasopharyngeal carcinoma- A restrospective review of patients less than thiny years of age: A report fnom Childrens Cancer Study Group. Cancer 1981; 47: 360-366.

25. Rahimo $M$, Rakowsky E, Barzilay J, Sidi J: Carcinoma of the nasopharynx. Cancer 1986; 58: 843-849.

26. Decker Do. Drefichman A, Al-Sarraf M. Reed ML: Chemotherapy for nasopharyngeal carcinoma. A tenyear experience, Cancer 1983; 52: 602-605.

27. Rossi A, Molinari R, Boracchi P, al.: Adjuvant chemotherapy with vincristine, cyclosphosphamide and doxorubicin after radiotherapy in local-regional nasopharyngeal cancer: Results of a 4 year multicenterrandomized swdy. J Clin Oncol 1988; 6: 1401-1410.

28. Choo $R$, Tannock $I$ : Chemotherapy for recurtent or metastatic carcinoma of the nasopharynx. A review of the Princess Margaret Hospital Experience. Cancer 1991; 68: 2120-2124.

29. Atichartakan V, Krainphibul $P$, Clongsuek $P$, at al.: Nasophraryngeal carcinoma: Result of treatment with cisdiarnminedichloroplatinum 115 Fluoruracil and tadiation theraphy. Int J Radiat Oncol Biol Phys 1988; 14: 461-469.

30. Fisch $R$ : The infratemporal fossa approach for nasopharyngeal lumors. Laryngoscope 1983; 93: 36-44. 